\title{
The reduced length of a polynomial with complex coefficients
}

by

\author{
A. Schinzel (Warszawa)
}

Dedicated to Wolfgang M. Schmidt on the occasion of his 75th birthday

For $P \in \mathbb{C}[x], P(x)=\sum_{i=0}^{d} a_{i} x^{d-i}=a_{0} \prod_{i=1}^{d}\left(x-\alpha_{i}\right)$, let

$$
\begin{gathered}
P^{*}(x)=\sum_{i=0}^{d} a_{i} x^{i}, \quad H(P)=\max _{0 \leq i \leq d}\left|a_{i}\right|, \\
L(P)=\sum_{i=0}^{d}\left|a_{i}\right|, \quad M(P)=\left|a_{0}\right| \prod_{i=1}^{d} \max \left\{1,\left|\alpha_{i}\right|\right\}, \\
l(P)=\inf L(P G), \quad \widehat{l}(P)=\min \left\{l(P), l\left(P^{*}\right)\right\},
\end{gathered}
$$

where $G$ runs through all monic polynomials in $\mathbb{C}[x]$. This notation is consistent with that of [3] and [4], since if $P \in \mathbb{R}[x]$ the above infimum coincides with $\inf L(P G)$, where $G$ runs through all monic polynomials in $\mathbb{R}[x]$. Some of the results about $l(P)$ stated in [1] and [3] for $P \in \mathbb{R}[x]$ carry over with essentially the same proof to $P \in \mathbb{C}[x]$. Thus we have

Proposition 1. Suppose that $\omega, \eta, \psi \in \mathbb{C},|\omega| \geq 1,|\eta|<1$. Then for every $Q \in \mathbb{C}[x]$,

(i) $l(\psi Q)=|\psi| l(Q)$,

(ii) $l(x+\omega)=1+|\omega|$,

(iii) if $T(x)=Q(x)(x-\eta)$, then $l(T)=l(Q)$,

(iv) $l(\bar{Q})=l(Q)$, where $\bar{Q}$ denotes the complex conjugate of $Q$.

Proposition 2. For all monic polynomials $P, Q$ in $\mathbb{C}[x]$, all $\eta \in \mathbb{C}$ with $|\eta|=1$ and all positive integers $k$,

(i) $\max \{l(P), l(Q)\} \leq l(P Q) \leq l(P) l(Q)$,

(ii) $M(P) \leq l(P)$,

2000 Mathematics Subject Classification: 12D99, 26C99.

Key words and phrases: reduced length, complex polynomial. 
(iii) $l(P(\eta x))=l(P(x))$,

(iv) $l\left(P\left(x^{k}\right)\right)=l(P(x))$.

Theorem 1. Let $P, Q \in \mathbb{C}[x], Q$ be monic and have all zeros on the unit circle. Then for all $m \in \mathbb{N}$,

$$
l\left(P Q^{m}\right)=l(P Q) .
$$

Theorem 2. If $P \in \mathbb{C}[x] \backslash \mathbb{C}$ is monic and has all zeros on the unit circle, then $\widehat{l}(P)=l(P)=2$, with $l(P)$ attained if all zeros are roots of unity and simple $(l(P)$ is attained means that $l(P)=L(Q)$, where $Q / P$ is a monic polynomial).

Theorems 1 and 2 correspond to Theorems 3 and 4 of [3], respectively. Also Theorem 6 of [3] extends to polynomials over $\mathbb{C}$, but the extension requires a different proof. We shall prove the following more general

Theorem 3. Let $P=P_{0} P_{1}$, where $P_{\nu} \in \mathbb{C}[x](\nu=1,2), L\left(P_{0}\right) \leq$ $2\left|P_{0}(0)\right|$ and $P_{1}$ is monic. Then

$$
l(P) \geq L\left(P_{0}\right)+\left(2\left|P_{0}(0)\right|-L\left(P_{0}\right)\right)\left(l\left(P_{1}\right)-1\right) .
$$

Corollary 1. If $P \in \mathbb{C}[x]$ and $L(P) \leq 2|P(0)|$, then

$$
l(P)=L(P) .
$$

Conversely, if $l(P)=L(P)$ and all coefficients of $P$ are real and positive, then $L(P) \leq 2 P(0)$.

Corollary 2. If $P(x)=(x-\alpha)(x-\beta)$, where $|\alpha| \geq|\beta| \geq 1$, then

$$
l(P) \geq 1+|\alpha|-|\beta|+|\alpha \beta|,
$$

with equality if $\alpha / \beta \in \mathbb{R}$ and either $\alpha / \beta<0$ or $|\beta|=1$.

One can prove that if $\alpha / \beta+\beta / \alpha \in \mathbb{R}$, the two cases given in Corollary 2 are the only ones for which there is equality in (1).

Corollary 3. If $P(x)=(x-\alpha)(x-\beta)$, where $|\alpha| \geq|\beta| \geq 1$, then

$$
l(P) \geq 2|\alpha|,
$$

with equality only possible if $|\beta|=1$. If moreover $\alpha / \beta \in \mathbb{R}$, then the equality really holds.

Corollary 4. Let $P=P_{0} P_{1}$, where $P_{\nu} \in \mathbb{C}[x](\nu=0,1), \operatorname{deg} P_{1} \geq 1$ and all zeros $z$ of $P_{\nu}$ satisfy $|z|>1$ for $\nu=0,|z|=1$ for $\nu=1$. If

$$
l\left(P_{0}\right)=L\left(P_{0}\right),
$$

then

$$
l(P) \geq 2 M(P) .
$$


It remains a problem whether (3) holds without the assumption (2). The following results point towards an affirmative answer.

Theorem 4. If $P \in \mathbb{C}[x] \backslash\{0\}$ has a zero $z$ with $|z|=1$, then

$$
L(P)>\sqrt{2} M(P), \quad l(P) \geq \sqrt{2} M(P) .
$$

Theorem 5. If $P(x)=(x-\alpha)(x-\beta)(x-1)$, where $\alpha, \beta$ are real and at least one of them is positive, then (3) holds.

The validity of (3) for all polynomials $P$ over $\mathbb{C}$ or over $\mathbb{R}$ with a zero on the unit circle is equivalent to the validity of a simpler inequality $L(P) \geq$ $2 M(P)$ for all polynomials $P$ over $\mathbb{C}$ or $\mathbb{R}$, respectively, with a zero on the unit circle. E. Dobrowolski has verified that the latter inequality is true for all such polynomials $P \in \mathbb{C}[x]$ of degree at most 4 .

I thank E. Dobrowolski and A. Dubickas for valuable criticism.

Proof of Theorem 3. Let $G$ be any monic polynomial in $\mathbb{C}[x]$ and let

$$
T(x)=P_{1}(x) G(x)=x^{n}+\sum_{i=1}^{n} b_{i} x^{n-i}, \quad b_{0}=1 .
$$

We have

$$
\sum_{i=1}^{n}\left|b_{i}\right|=L(T)-1 \geq l\left(P_{1}\right)-1
$$

Now, let

$$
P_{0}(x)=\sum_{i=0}^{d} a_{i} x^{d-i}
$$

We have

$$
\begin{aligned}
Q(x) & =P(x) G(x)=P_{0}(x) T(x)=\left(\sum_{i=0}^{d} a_{i} x^{d-i}\right)\left(\sum_{i=0}^{n} b_{i} x^{n-i}\right) \\
& =\sum_{j=0}^{d+n}\left(\sum_{i=0}^{\min \{j, d\}} a_{i} b_{j-i}\right) x^{d+n-j} .
\end{aligned}
$$

Now

$$
\left|\sum_{i=0}^{\min \{j, d\}} a_{i} b_{j-i}\right| \geq \begin{cases}\left|a_{j}\right|-\sum_{i=0}^{j-1}\left|a_{i}\right|\left|b_{j-i}\right| & \text { for } j \leq d, \\ \left|a_{d}\right|\left|b_{j-d}\right|-\sum_{i=0}^{d-1}\left|a_{i}\right|\left|b_{j-i}\right| & \text { for } j>d\end{cases}
$$


hence

$$
\begin{aligned}
L(Q) & =\sum_{j=0}^{d+n}\left|\sum_{i=0}^{\min \{j, d\}} a_{i} b_{j-i}\right| \\
& \geq \sum_{j=0}^{d}\left|a_{j}\right|-\sum_{j=1}^{d} \sum_{i=0}^{j-1}\left|a_{i}\right|\left|b_{j-i}\right|+\sum_{j=d+1}^{d+n}\left|a_{d}\right|\left|b_{j-d}\right|-\sum_{j=d+1}^{d+n} \sum_{i=0}^{d-1}\left|a_{i}\right|\left|b_{j-i}\right| \\
& =L\left(P_{0}\right)+\left|a_{d}\right|(L(T)-1)-\sum_{j=1}^{d+n} \sum_{i=0}^{\min \{j-1, d-1\}}\left|a_{i}\right|\left|b_{j-i}\right| \\
& =L\left(P_{0}\right)+\left|P_{0}(0)\right|(L(T)-1)-\left(L\left(P_{0}\right)-\left|P_{0}(0)\right|\right)(L(T)-1)
\end{aligned}
$$

and since, by the assumption, $\left|2 P_{0}(0)\right|-L\left(P_{0}\right) \geq 0$ it follows from (4) that

$$
L(Q) \geq L\left(P_{0}\right)+\left(2\left|P_{0}(0)\right|-L\left(P_{0}\right)\right)\left(l\left(P_{1}\right)-1\right) .
$$

Proof of Corollary 1. In order to obtain the first statement we take $P_{1}=1$ in Theorem 3 . In order to obtain the second statement, let

$$
P(x)=\sum_{i=0}^{d} a_{i} x^{d-i}, \quad \eta=\min _{0<i \leq d} \frac{a_{i}}{a_{i-1}}
$$

and assume that $L(P)>2 a_{d}$. Then

$L(P(x)(x-\eta))=a_{0}+\sum_{i=1}^{d}\left(a_{i}-a_{i-1} \eta\right)+a_{d} \eta=L(P)-\left(L(P)-2 a_{d}\right) \eta<L(P)$.

Proof of Corollary 2. Taking $P_{0}=x-\alpha, P_{1}=x-\beta$ in Theorem 3 and using Corollary 1 to evaluate $l\left(P_{1}\right)$ we obtain (1). If $\alpha / \beta \in \mathbb{R}$ and $\alpha / \beta<0$ we have $L(P)=1+|\alpha+\beta|+|\alpha \beta|=1+|\alpha|-|\beta|+|\alpha \beta|$, hence $l(P)=L(P)$. If $\alpha / \beta \in \mathbb{R}, \alpha / \beta>0$ and $|\beta|=1$, then for $|\alpha|=1$ we have $l(P)=2=1+|\alpha|-|\beta|+|\alpha \beta|$ by Theorem 2. For $|\alpha|>1$ we infer from the divisibility

that

$$
P \mid x^{n+1}-\frac{\alpha^{n+1}-\beta^{n+1}}{\alpha^{n}-\beta^{n}} x^{n}+(\alpha \beta)^{n} \frac{\alpha-\beta}{\alpha^{n}-\beta^{n}}
$$

$$
\begin{aligned}
l(P) & \leq 1+\lim _{n \rightarrow \infty}\left|\frac{\alpha^{n+1}-\beta^{n+1}}{\alpha^{n}-\beta^{n}}\right|+\lim _{n \rightarrow \infty}\left|\alpha^{n} \frac{\alpha-\beta}{\alpha^{n}-\beta^{n}}\right|=1+|\alpha|+|\alpha-\beta| \\
& =1+|\alpha \beta|+|\alpha|-|\beta|,
\end{aligned}
$$

hence again $l(P)=1+|\alpha|-|\beta|+|\alpha \beta|$.

Proof of Corollary 3. The first part of the corollary follows from the first part of Corollary 2 and the identity

$$
1+|\alpha|-|\beta|+|\alpha \beta|-2|\alpha|=(|\alpha|-1)(|\beta|-1) .
$$

The second part follows from the second part of Corollary 2. 
Proof of Corollary 4. Multiplying $P_{0}$ by a constant we may assume that $P_{1}$ is monic. If $l\left(P_{0}\right) \geq 2 M\left(P_{0}\right)$ we have

$$
l(P) \geq l\left(P_{0}\right) \geq 2 M\left(P_{0}\right)=2 M(P) .
$$

If $l\left(P_{0}\right)<2 M\left(P_{0}\right)=2\left|P_{0}(0)\right|$ we have $L\left(P_{0}\right)<2\left|P_{0}(0)\right|$ by $(2)$, and since, by Theorem $2, l\left(P_{1}\right)=2$, Theorem 3 gives

$$
l(P) \geq L\left(P_{0}\right)+2\left|P_{0}(0)\right|-L\left(P_{0}\right)=2 M\left(P_{0}\right)=2 M(P) .
$$

For the proof of Theorem 4 we need

Lemma 1. If $P \in \mathbb{C}[x]$ has at least one zero $\varepsilon$ with $|\varepsilon|=1$, then

$$
L(P) \geq 2 H(P) \text {. }
$$

Proof. Let $P(x)=(x-\varepsilon) \sum_{i=0}^{d-1} b_{i} x^{d-i-1}$, where $|\varepsilon|=1$. We have

$$
P(x)=\sum_{i=0}^{d}\left(b_{i}-\varepsilon b_{i-1}\right) x^{d-i}, \quad \text { where } \quad b_{-1}=b_{d}=0 .
$$

Assuming that

$$
H(P)=\left|b_{j}-\varepsilon b_{j-1}\right|
$$

we have

$$
\begin{aligned}
L(P) & =\sum_{i=0}^{j-1}\left|b_{i}-\varepsilon b_{i-1}\right|+H(P)+\sum_{i=j+1}^{d}\left|b_{i}-\varepsilon b_{i-1}\right| \\
& \geq \sum_{i=0}^{j-1}\left(\left|b_{i}\right|-\left|b_{i-1}\right|\right)+H(P)+\sum_{i=j+1}^{d}\left(\left|b_{i-1}\right|-\left|b_{i}\right|\right) \\
& \geq\left|b_{j-1}\right|+H(P)+\left|b_{j}\right| \geq 2 H(P) .
\end{aligned}
$$

Proof of Theorem 4. Let $P(x)=\sum_{i=0}^{d} a_{i} x^{d-i}$. Then

$$
\|P\|^{2}:=\sum_{i=0}^{d}\left|a_{i}\right|^{2} \leq H(P) L(P)
$$

and, by Lemma 1 ,

$$
L(P)^{2} \geq 2\|P\|^{2} .
$$

However, $P$ has at least two non-zero coefficients, hence by Theorem 40 of $[2]$,

$$
\|P\|>M(P) .
$$

The first inequality of Theorem 4 follows from (5) and (6). Hence for every monic $G$ in $\mathbb{C}[x], L(P G)>\sqrt{2} M(P G) \geq \sqrt{2} M(P)$, which implies $l(P) \geq$ $\sqrt{2} M(P)$.

For the proof of Theorem 5 we need five lemmas. 
Lemma 2. If $k>l \geq 1$, the function

$$
g(x)=\frac{x^{k}-1}{x^{l}-1}
$$

is strictly increasing for $x>1$.

Proof. We have

$$
g^{\prime}(x)=\frac{x^{l-1} f(x)}{\left(x^{l}-1\right)^{2}}, \quad \text { where } \quad f(x)=(k-l) x^{k}-k x^{k-l}+l .
$$

Now $f(1)=0, f^{\prime}(x)=(k-l) k\left(x^{k-1}-x^{l-1}\right)>0$ for $x>1$, hence $f(x)>0$ and $g(x)$ is strictly increasing.

Lemma 3. Let $\alpha, \beta \in \mathbb{R}$ and $\alpha \geq \beta>1, k>l \geq 1$,

$$
D(k, l ; \alpha, \beta)= \begin{cases}\left(\alpha^{k}-1\right)\left(\beta^{l}-1\right)-\left(\alpha^{l}-1\right)\left(\beta^{k}-1\right) & \text { if } \alpha \neq \beta, \\ k \alpha^{k-1}\left(\alpha^{l}-1\right)-l \alpha^{l-1}\left(\alpha^{k}-1\right) & \text { if } \alpha=\beta .\end{cases}
$$

Then

$$
D(k, l ; \alpha, \beta)>0 .
$$

Proof. For $\alpha>\beta$ we have, in the notation of Lemma 2,

$$
D(k, l ; \alpha, \beta)=\left(\beta^{k}-1\right)\left(\beta^{l}-1\right)(g(\alpha)-g(\beta)),
$$

and (7) follows from Lemma 2. For $\alpha=\beta$ we have

$$
D(k, l ; \alpha, \beta)=\alpha^{l-1} f(\alpha),
$$

and (7) follows from the inequality $f(x)>0$ for $x>1$ established in the proof of Lemma 2.

Lemma 4. If $P(x)=(x-\alpha)(x-\beta)(x-1), \alpha \geq \beta>1$, then every monic polynomial divisible by $P$ with at most four non-zero coefficients is of the form

$$
x^{m}+a x^{n}+b x^{p}+c,
$$

where $m>n>p>0$ and

$$
a=-\frac{D(m, p ; \alpha, \beta)}{D(n, p ; \alpha, \beta)}, b=\frac{D(m, n ; \alpha, \beta)}{D(n, p ; \alpha, \beta)}, c=-(\alpha \beta)^{p} \frac{D(m-p, n-p ; \alpha, \beta)}{D(n, p ; \alpha, \beta)} .
$$

Proof. The above values of $a, b, c$ are obtained by solving the systems of linear equations

$$
\begin{aligned}
& \alpha^{m}+a \alpha^{n}+b \alpha^{p}+c=0, \\
& \beta^{m}+a \beta^{n}+b \beta^{p}+c=0, \\
& 1+a+b+c=0
\end{aligned}
$$


if $\alpha>\beta$, and

$$
\begin{aligned}
& m \alpha^{m-1}+n a \alpha^{n-1}+p b \alpha^{p-1}=0, \\
& \alpha^{m}+a \alpha^{n}+b \alpha^{p}+c=0, \\
& 1+a+b+c=0
\end{aligned}
$$

otherwise, with the determinant $D(n, p ; \alpha, \beta)$, which is non-zero by virtue of Lemma 3.

Lemma 5. If $r$ is a positive integer, and $t, x \geq 1$, then

$$
\frac{t^{r} x^{r+1}}{1+t+\cdots+t^{r-1}} \geq t x^{2}-1
$$

Proof. For $r=1$ the inequality is clear. For $r \geq 2$ let $t_{0}$ be the unique positive root of the equation

$$
h(t)=2+2 t+\cdots+2 t^{r-1}-(r+1) t^{r-1}=0 .
$$

We have

$$
1<t_{0}<\frac{r+1}{r-1}
$$

since $h(1)=r-1>0$ and $h\left(\frac{r+1}{r-1}\right)=1-r<0$.

Put

$$
x_{0}(t)=\left(\frac{2+2 t+\cdots+2 t^{r-1}}{(r+1) t^{r-1}}\right)^{1 /(r-1)} .
$$

The function

$$
F(t, x)=t^{r} \frac{x^{r+1}}{1+\cdots+t^{r-1}}-t x^{2}+1
$$

is decreasing for $x<x_{0}(t)$ and increasing for $x>x_{0}(t)$. If $t<t_{0}$ we have $x_{0}(t)>1$, if $t \geq t_{0}$ we have $x_{0}(t) \leq 1 \leq x$. Therefore, for $t \geq t_{0}$,

$$
F(t, x) \geq F(t, 1)=\frac{1}{1+\cdots+t^{r-1}}>0 .
$$

For $t<t_{0}$ we have

$$
\begin{aligned}
F(t, x) & \geq F\left(t, x_{0}(t)\right)=t^{r} \frac{x_{0}(t)^{r+1}}{1+\cdots+t^{r-1}}-t x_{0}(t)^{2}+1 \\
& =t x_{0}(t)^{2}\left(\frac{t^{r-1}}{1+\cdots+t^{r-1}} x_{0}(t)^{r-1}-1\right)+1=t x_{0}(t)^{2}\left(\frac{2}{r+1}-1\right)+1 \\
& =1-\frac{r-1}{r+1} t x_{0}(t)^{2} .
\end{aligned}
$$

Assuming that the right-hand side is negative we obtain

$$
t x_{0}(t)^{2}>\frac{r+1}{r-1}
$$


thus

$$
t^{(r-1) / 2} \cdot 2 \cdot \frac{1+\cdots+t^{r-1}}{(r+1) t^{r-1}}>\left(\frac{r+1}{r-1}\right)^{(r-1) / 2}
$$

and

$$
2\left(t^{-(r-1) / 2}+\cdots+t^{(r-1) / 2}\right)>\left(\frac{r+1}{r-1}\right)^{(r-1) / 2}(r+1) .
$$

The function $t^{-1}+t$ is increasing for $t \geq 1$ and so is $t^{-(r-1) / 2}+\cdots+t^{(r-1) / 2}$. Hence $t<t_{0}$ implies

$$
2 \frac{t_{0}^{r}-1}{t_{0}-1} t_{0}^{-(r-1) / 2}=2\left(t_{0}^{-(r-1) / 2}+\cdots+t_{0}^{(r-1) / 2}\right)>\left(\frac{r+1}{r-1}\right)^{(r-1) / 2}(r+1),
$$

thus, by the definition of $t_{0}$,

$$
(r+1) t_{0}^{(r-1) / 2}>\left(\frac{r+1}{r-1}\right)^{(r-1) / 2}(r+1)
$$

and

$$
t_{0}>\frac{r+1}{r-1}
$$

contrary to $(10)$.

LEMMA 6. If $\alpha \geq \beta>1$, then in the notation of Lemma 4,

$$
a<0, \quad b \geq \alpha \beta-1, \quad c<0 \text {. }
$$

Proof. By Lemma 3 we have in this case $a<0, b>0, c<0$, hence

$$
\alpha^{m}+|b| \alpha^{p}=|a| \alpha^{n}+|c|<(|a|+|c|) \alpha^{n}
$$

and

$$
b+1=|a|+|c|>\alpha^{n-m} \geq \alpha \beta,
$$

unless $m=n+1$.

Consider first the case $\alpha>\beta$. Assuming $m=n+1$ we infer from (7) and (8) that

$$
\alpha-\beta+b\left(\alpha^{p-n}-\beta^{p-n}\right)+c\left(\alpha^{-n}-\beta^{-n}\right)=0,
$$

and since $\alpha^{p-n}-\beta^{p-n}<0$ and $c\left(\alpha^{-n}-\beta^{-n}\right)>0$,

$$
b \geq \frac{\alpha-\beta}{\beta^{p-n}-\alpha^{p-n}} .
$$

Putting $n-p=r, \alpha=t \beta$ we obtain $t \geq 1$, and by Lemma 5 ,

$$
b \geq \frac{t^{r} \beta^{r-1}}{1+\cdots+t^{r-1}} \geq t \beta^{2}-1=\alpha \beta-1 .
$$

Consider now the case $\alpha=\beta$. Then by the case already proved,

$$
b=\lim _{\beta \rightarrow \alpha-0} \frac{D(m, n ; \alpha, \beta)}{D(n, p ; \alpha, \beta)} \geq \lim _{\beta \rightarrow \alpha-0}(\alpha \beta-1)=\alpha^{2}-1 .
$$


Proof of Theorem 5. Let $|\alpha| \geq|\beta|$. If $|\alpha|<1$, then by Proposition 1(ii), (iii),

$$
l(P)=l(x-1)=2=2 M(P) .
$$

If $|\beta|<1 \leq|\alpha|$, then by Proposition 1 (iii) and by Corollary 3,

$$
l(P)=l((x-\alpha)(x-1))=2|\alpha|=2 M(P) .
$$

If $\beta=1$ the same is true by Theorem 1 and Corollary 3. If $|\beta| \geq 1$ and $\alpha / \beta<0$, then by Corollary 2 ,

$$
l(P)=l((x-\alpha)(x-\beta))=L((x-\alpha)(x-\beta)),
$$

hence, by Corollary 4,

$$
l(P) \geq 2 M(P) .
$$

Finally, if $|\beta| \geq 1, \beta \neq 1$ and $\alpha / \beta>0$, then by the assumption that at least one of $\alpha, \beta$ is positive we have $\alpha \geq \beta>1$ and by Theorem 1 of [3],

$$
l(P)=\inf _{Q \in S_{3}(P)} L(Q),
$$

while, by Lemma 4 , each element $Q$ of $S_{3}(P)$ is of the form $Q=x^{m}+$ $a x^{n}+b x^{p}+c$, where $a, b, c$ are given by the formulae of Lemma 4 . Now, by Lemma 6 ,

$$
a<0, \quad b \geq \alpha \beta-1, \quad c<0,
$$

hence $L(Q)=L(Q)+Q(1)=2(1+b) \geq 2 \alpha \beta=2 M(P)$.

Note added in proof. Concerning Proposition 2(ii) E. Dobrowolski has observed that if $M(P)>\left|a_{0}\right|$, then $l(P)>M(P)$. Indeed, then for every monic $Q$ we have $L(P Q) \geq$ $\left|a_{0}\right|+\sqrt{\|P Q\|^{2}-\left|a_{0}\right|^{2}}>\left|a_{0}\right|+\sqrt{M(P Q)^{2}-\left|a_{0}\right|^{2}} \geq\left|a_{0}\right|+\sqrt{M(P)^{2}-\left|a_{0}\right|^{2}}$, hence $l(P) \geq$ $\left|a_{0}\right|+\sqrt{M(P)^{2}-\left|a_{0}\right|^{2}}>M(P)$.

\section{References}

[1] A. Dubickas, Arithmetical properties of powers of algebraic numbers, Bull. London Math. Soc. 38 (2006), 70-80.

[2] A. Schinzel, Polynomials with Special Regard to Reducibility, Cambridge Univ. Press, 2000 .

[3] —, The reduced length of a polynomial with real coefficients, in: Selecta, Vol. 1, Eur. Math. Soc., Zürich, 2007, 658-691.

[4] - The reduced length of a polynomial with real coefficients II, Funct. Approx. Comment. Math. 37 (2007), 445-459.

Institute of Mathematics

Polish Academy of Sciences

Śniadeckich 8, P.O. Box 21

00-956 Warszawa, Poland

E-mail: schinzel@impan.gov.pl 